\section{Laser Fusion Los Alamos} Experiment

On March 4, 1977 the press services reported a "key step" toward fusion at the Los Alamos laboratory of the United States Energy Research and Development Administration (USERDA). The experiment which led to the news release was the following:

Glass microbalioons, approximately $100 \mu \mathrm{m}$ in diameter with $1 \mu \mathrm{m}$ walls, containing deuterium-tritium gas at $10 \mathrm{~atm}$ pressure, were used as targets in a new $\mathrm{CO}_{2}$ laser facility. Two beams delivered, simultaneously, to opposite sides of the target, an energy of $250 \mathrm{~J}$ each, in a $1 \mathrm{~ns}$ pulse injecting thus an incident power of 0.5 TW. The experiments produced $10^{4}$ to $10^{5}$ fusion neutrons. The importance of these experiments does not lie in the number of fusion neutrons produced. Neutrons have been produced with glass lasers in various laboratories, the record at present being about $10^{\circ}$ neutrons with a 2 TW glass laser at Livermore (also a USERDA laboratory). While the power delivered to the target was a record for $\mathrm{CO}_{2}$ lasers, that was also of secondary importance.

The significance of the Los Alamos measurements is that the $\mathrm{CO}_{2}$ laser produced the same neutron yields as those achieved earlier with glass lasers at the same power level. This new result, together with various experimental and theoretical information accumulated in the past year, indicates the the $\mathrm{CO}_{2}$ laser may be as suited for laser-fusion experiments as the Nd: glass laser. It had been legend that the $\mathrm{CO}_{2}$ laser is unsuited for such experiments. The $10.6 \mu \mathrm{m}$ (far infrared) $\mathrm{CO}_{2}$ wavelength was assumed, on the basis of naive models, to couple much more poorly to a target than the $1.06 \mu \mathrm{m}$ (near infrared) Nd: glass wavelength.

The advantages of high efficiency and a gas lasing medium motivated the original Los Alamos decision to promote $\mathrm{CO}_{2}$ laser development. The efficiency for conversion of electrical energy to laser energy is $\leq 3 \%$ for $\mathrm{CO}_{2}$ as opposed to $\leq 0.1$ for glass lasers - a decisive advantage.

\section{ESA's Geostationary Satellite Operating Despite Launch Problems}

There are strong hopes that the mission of GEOS, the satellite of the European Space Agency that was to be the world's first all-scientific geostationary satellite will be much more successful than had been feared when it was realized that the launcher had failed to place it in the correct parking orbit. A fault in the separation of the second and third stages was the cause of the error. It had been intended to transfer GEOS from its parking orbit into a geostationary orbit $36000 \mathrm{~km}$ above the equator from where it would have been permanently in view of the Odenwald ground station in Germany built specially for receiving data from the satellite. This was no longer possible and an immediate problem facing the Agency was to decide quickly what new orbit to choose, as in the parking orbit, the satellite was passing through high radiation belts and degradation of the solar cells was marked. The output power dropped from $135 \mathrm{~W}$ to $105 \mathrm{~W}$ in four days and any further drop would have meant that there would have been insufficient power to fire the motors.

The orbit decided upon was a highly eccentric orbit with an apogee of $36000 \mathrm{~km}$ and a perigee of $2000 \mathrm{~km}$ which brings it in sight of the ground station in Germany for seven hours per day, when data can be taken in real time. It is hoped that a second station can be opened in the United States which will be able to supplement this receiving period. placed in its final orbit there was no further degradation of the power system and the experiments were all switched on successfully and began
In the first weeks since GEOS was taking data. From a technological stand-point it seems clear that the satellite works well and much useful data should be collected. It is difficult at the moment to forecast its life but it should continue to operate for at least the four to six months that was its minimum design life-time even if it cannot survive as long as it would have done in its design orbit.

The main purpose of GEOS is to study the magnetosphere, and its pay load consists of seven experiments involving eleven research centres in eight countries. It has been constructed so as to be "superclean" in the magnetic, electric and eletromagnetic regimes. The equipment it carries includes a number of magnetometers, electrostatic analyser, aerials for electric field measurements both $D C$ and $A C$, and particle distribution analysers as well as the transmission system able to handle $100 \mathrm{k}$ bits/s. GEOS was also intended to act as the reference satellite for the International Magnetospheric Study (IMS), a three-year research programme in which Soviet, American and Japanese spacecraft are also participating together with flights of sounding rockets, balloons and aircraft.

Discussions are currently going on to decide whether to continue with the original plan of putting the qualification model into a geostationary orbit in 1979 under the code name Geosare or whether to use the model to repeat the original mission at an earlier date. ESA is due to bring into operation two further geostationary satellites in the near future. In mid-June the telecommunication satellite OTS is scheduled for lauching and in mid-September the meteorological satellite Meteosat.

\section{Post-doctoral Position in Surface Sciences}

The Belgian interuniversity program IRIS (Institute for Research in Interface Sciences) shared between the Universities of Namur and Mons, Belgium, invites applications from postdoctoral physicists or sabbatical visiting scientists in the field of surface sciences to work in one or several of the following fields :

1) gas-surface interactions

2) thin films nucleation and growth

3) extended radiation damage in metals and semi-conductors.

Modern experimental facilities include XPS-UPS, LEED-AUGER, CELS, Infrared Absorption Reflection Spectroscopy. Preference will be given to surface physicists with previous experience in the above fields.

Starting dates: September 1977 in Mons and January 1978 in Namur.

Duration and level of appointment open to negociations. Send application with detailed resume to either: Prof. L. Laude, Solid State Physics Laboratory, Université de l'Etat à Mons, 7000 Mons or Prof. A. A. Lucas, Physics Department, Facultés Universitaires de Namur, 5000 Namur. 